\title{
Visualization of Meiotic Chromosomes by Negative-Stain Transmission Electron Microscopy: Preparation and Staining Methods Using Common Staining Reagents
}

\author{
M. J. de la Cruz, S. J. Lockett, and K. Nagashima \\ Electron Microscopy Facility/Image Analysis Laboratory, SAIC-Frederick, Inc., NCI-Frederick, \\ P.O. Box B, Frederick, MD 21702-1201
}

Whole-mount chromosome spreads have long been used for the examination of meiotic chromosomes via light and electron microscopy. The prevailing staining method for spreads, particularly of mouse spermatocyte samples, involves the use of silver nitrate because it is designed for both light and electron microscopy examination [1]. Since our focus is with electron microscopy, we have skipped the step for light microscopy preparation in favor of a faster procedure involving one of two common negative-staining reagents, UA (uranyl acetate) and PTA (phosphotungstic acid). PTA has been known to stain chromosomes for electron microscopy [2]. As a core facility for the National Institutes of Health, our laboratory must maintain the highest standards of scientific quality while maximizing sample turnover rates. We have standardized methods for (1) rapid processing of chromosome spreads and (2) an optimal staining procedure to quickly visualize meiotic chromosomes by negative-stain TEM.

Mouse spermatocytes were prepared for whole-mount chromosome spreading. A droplet of the spermatocytes in cell suspension was placed at the center of a formvar-coated glass slide, fixed in $2 \%$ glutaraldehyde, and allowed to air-dry. The formvar film was separated from the slide and placed onto 3-mm copper mesh TEM grids. These grids were then stained with either 1\% UA or $1 \%$ PTA ( $\mathrm{pH}$ 7.0). This grid-staining method is easier to perform than the slide-staining method commonly employed with silver nitrate [3].

Images of both UA- and PTA-stained samples revealed the body of the chromosomes, known as the lateral element. The chromosomes stained with UA did not show as much detail as those stained with PTA. In addition, the central element (inner segment within the lateral element) was easily seen in the PTA-stained chromosome, unlike the UA-stained sample. We had also stained the samples with $0.33 \%$ silver nitrate using the same method with nickel grids, but we could not produce comparable results (data not shown). Thus, the PTA stain is superior to the UA stain in negativestain studies of chromosome ultrastructure. These methods are most useful to investigators who do not require images at the light microscopy level prior to electron microscopy examination.

References

[1] M. E. Dresser and M. J. Moses, Exp Cell Res 121 (1979) 416.

[2] S. J. Counce and G. F. Meyer, Chromosoma 44 (1973) 231.

[3] C. Barlow, et al., Development 125 (1998) 4007.

[4] We gratefully acknowledge Dr. S. Sharan and Dr. S. Kuznetsov of the Mouse Cancer Genetics Program, National Cancer Institute, for chromosome spread samples and support.

[5] Funded by NCI Contract N01-CO-12400. 

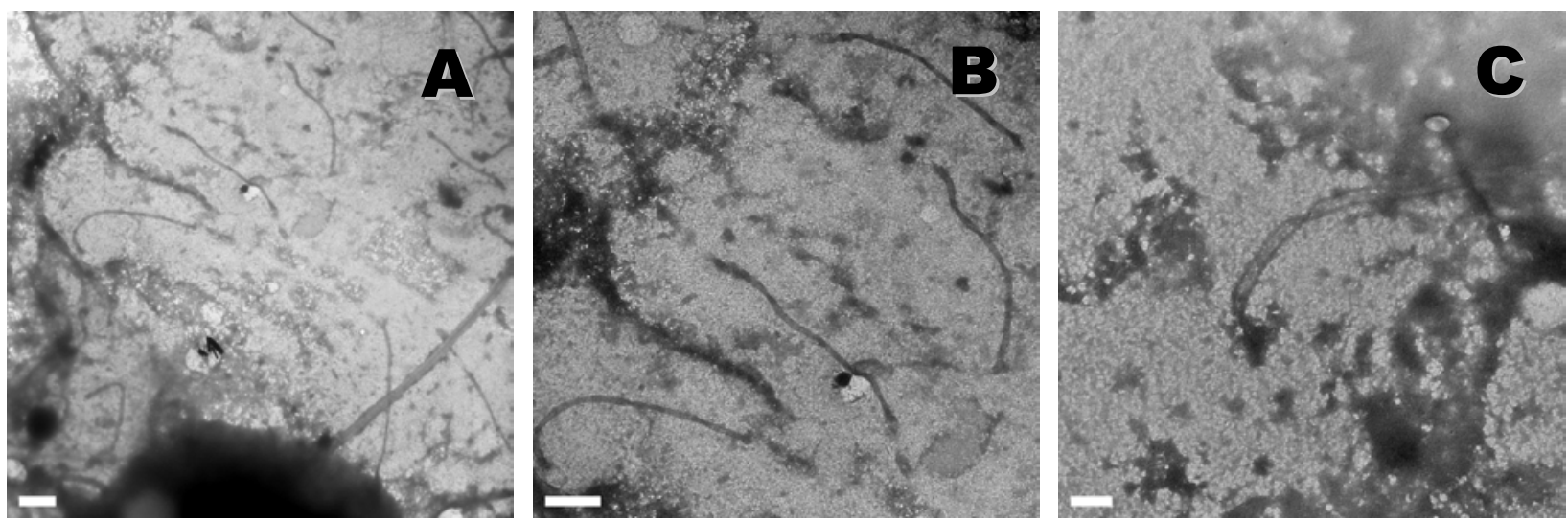

Fig. 1. Chromosome spread stained with 1\% UA. (A) Chromosomes appear as the thin line segments. Scale bar, $2 \mu \mathrm{m}$. (B) Higher magnification. Scale bar, $2 \mu \mathrm{m}$. (C) Another area at high magnification shows a twisting of the lateral element of a chromosome. Scale bar, $500 \mathrm{~nm}$.
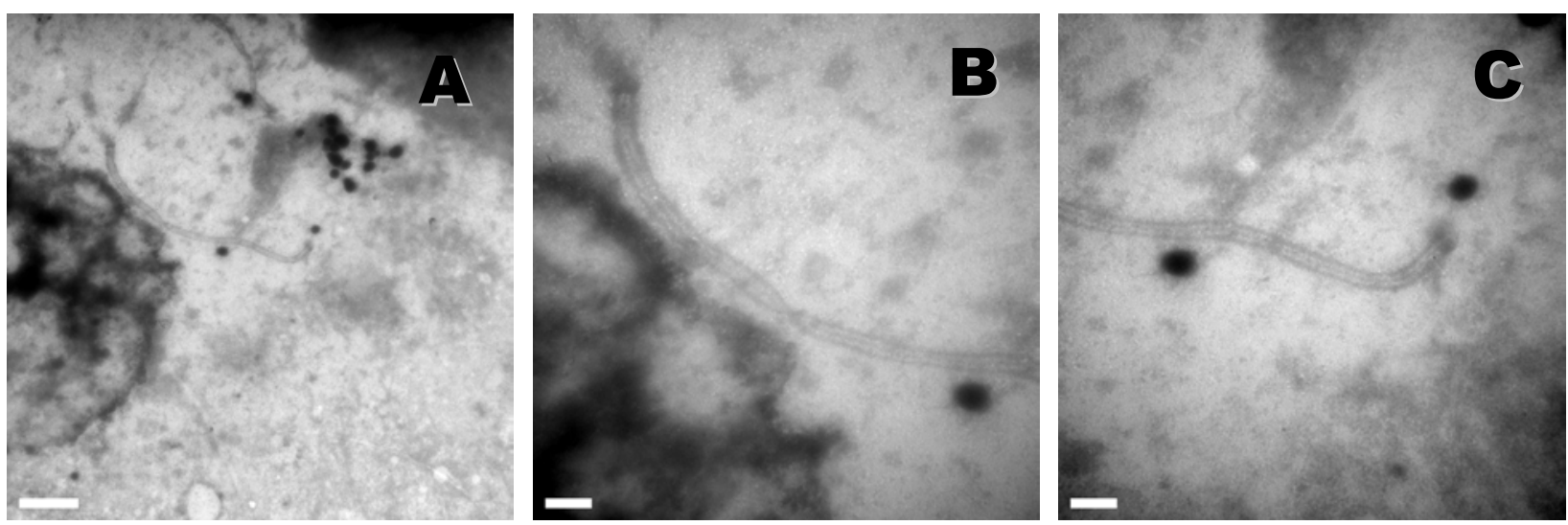

Fig. 2. Chromosome spread stained with 1\% PTA. (A) Two chromosomes appear at the top of the image. Scale bar, $2 \mu \mathrm{m}$. (B) Higher magnification of the chromosome towards the center of the previous image. The central element can be seen, as well as twisting of the chromosome. Scale bar, $500 \mathrm{~nm}$. (C) Right end of the chromosome continued from the previous image. Scale bar, $500 \mathrm{~nm}$. 